\title{
Characterization and Antibiotic Sensitivity Profile of Bacteria Isolated from Patients with Respiratory Tract Infections in Bangladesh
}

\author{
Shukla Promite ${ }^{1}$, Sajal K. Saha ${ }^{2}$, Sunjukta Ahsan ${ }^{1}$ and Marufa Zerin Akhter ${ }^{1}$ \\ ${ }^{1}$ Department of Microbiology, University of Dhaka, Dhaka, Bangladesh \\ ${ }^{2}$ Department of General Practice, Monash University, Building 1, 270 Ferntree Gully Road, Notting \\ Hill VIC 3168, Australia
}

(Received: October 08, 2017; Accepted: December 15, 2017; Published (web): December 23, 2017)

\begin{abstract}
The study was aimed to characterize bacterial isolates from respiratory tract infections (RTI) and investigate their antibiotic sensitivity profile. Selective media and biochemical tests were used to characterize 40 bacterial isolates. Antibiotic sensitivity testing was conducted using Kirby-Bauer disc diffusion method. About $42.5 \%$ (17) RTI patients were infected by Klebsiella pneumoniae, 30\% (12) by Escherichia coli and 27.5\% (11) by Pseudomonas aeruginosa with no significant gender variation (p-value $<0.578$ ). Overall, $47 \%$ (out of 20 ) antibiotics were sensitive, whereas $48 \%$ were resistant. Surprisingly, $18 \%$ P. aeruginosa and $20 \%$ K. pneumoniae were carbapenem-resistant and 4 out of 7 cephalosporin antibiotics were highly resistant irrespective of pathogens. E. coli showed better sensitivity to nitrofurantoin (78\%) and levofloxacin (89\%), while K. pneumoniae was insensitive to cotrimoxazole (88\%), gentamycin $(77 \%)$ and piperacillin/tazobactam $(66 \%)$. On the other hand, P. aeruginosa did not respond to $P$. aeruginosa to nalidixic acid $(60 \%)$ and ciprofloxacin $(60 \%)$. This study concludes that nitrofurantoin, levofloxacin, cotrimoxazole, gentamycin and piperacillin/tazobactam antibiotics could be better alternative in treating bacterial RTIs.
\end{abstract}

Key words: Antibiotic sensitivity, bacterial pathogens, RTIs, Bangladesh.

\section{INTRODUCTION}

Antibiotic resistance (AR) is a global public health concern. ${ }^{1}$ By 2050 , it is estimated that drugresistant infections will cause 10 million death and global economic loss of US $\$ 60-100$ trillion if AR continues to rise at a similar pace as now. ${ }^{2}$ The rising bacterial resistance is common among Respiratory Tract Infections (RTI). The causative pathogens of RTIs are Streptococcus pneumoniae, Klebsiella pneumoniae, Pseudomonas aeruginosa and Escherichia coli. ${ }^{1,3,4}$ RTIs are one of the significant causes of global morbidity and mortality as well. ${ }^{5}$ Now-a-days in appropriate prescribing of antibiotics in RTIs has become a common malpractice by many prescribers. ${ }^{6,7}$ This practice further magnifies the emergence of antibiotic-resistant bacterial strains in addition to increased adverse effect, treatment cost, resource use and consultation with doctors. ${ }^{6-8}$

Correspondence to: Shukla Promite

E-mail: spromite27@ gmail.com; Phone no: 01557258205

Dhaka Univ. J. Pharm. Sci. 16(2): 235-244, 2017 (December)
The rise of AR in Bangladesh is probably due to its difficulties in establishing bacterial etiology at the time of prescribing antibiotic in RTIs. ${ }^{9,10}$ Moreover, context and disease-specific surveillance data of antimicrobial sensitivity are limited in Bangladesh, ${ }^{10}$ although this information has paramount importance to develop local and national antibiotic guidelines. RTIs specific antibiotic guidelines are very important in this context to reduce over and imprudent prescription of antibiotics by prescribers at all levels from primary to tertiary care.

Taking this into consideration, this study was designed mainly with two aims: i) to investigate the distribution of bacterial pathogens among RTI patients and ii) to investigate their antibiotic sensitivity profile. This study contained helpful information for the clinicians, pharmacists and health policymakers about sensitive antibiotics for treating bacterial RTIs in Bangladesh. 


\section{MATERIALS AND METHODS}

Different culture media and antibiotic disks were used for growth and characterization of the bacterial isolates and to test their sensitivity to antibiotics. Nutrient agar (Merck, Germany), Trypticase Soy broth (Merck, Germany), Mueller Hinton Broth (Merck, Germany), Mueller Hinton agar (Merck, Germany), MacConkey agar (Merck, Germany), Eosin Methylene Blue agar (Merck, Germany), Cetrimide agar(Merck, Germany), Kligler's iron agar (KIA) (Merck, Germany), Motility Indole Ornithine agar (MIO) (Merck, Germany), Simmons Citrate Agar(Merck, Germany), MR-VP (Merck, Germany), Catalase test (Merck, Germany) and commercial antibiotic discs (Merck KGaA, Darmstadt, Germany) were used in this experiment for biochemical characterization and antibiotic sensitivity of the isolated bacterial species.

Sample collection. The sputum samples were collected from clinically diagnosed RTI infected patients attending in a private hospital in Bangladesh. The samples were collected for the period of 5 months between February to June in 2016. The research was carried out at the Department of Microbiology, University of Dhaka, Bangladesh. The population studied was a heterogeneous with different age groups (from 6 months to $>70$ years). A total of 40 sputum samples were collected from male $(n=23)$ and female $(n=17)$ patients suffered from RTIs. Demographic information was collected via the counselors after obtaining informed consent from each patient with the assurance that all information obtained would be treated confidentially. According to Gradwohl's clinical laboratory methods and diagnosis, ${ }^{11}$ early morning sputum samples were collected in the autoclaved test tubes where nutrient agar medium was allowed to harden in a slanted position. For the preparation of nutrient agar, $0.5 \mathrm{~g}$ peptone, $0.3 \mathrm{~g}$ beef extract, $0.5 \mathrm{~g} \mathrm{NaCl}, 1.5 \mathrm{~g}$ agar were mixed with $100 \mathrm{ml}$ distilled water in an Erlenmeyer flask ( $\mathrm{pH}$ at 7.2). The media was autoclaved at $121^{\circ} \mathrm{C}$ for 30 minutes. After autoclaving, $5 \mathrm{ml}$ of media was dispensed into each tube and allowed to solidify in a slanted position.
Samples were collected in this media and transported to the laboratory.

Bacterial culture. Samples were inoculated onto nutrient agar plate followed by incubation at $37^{\circ} \mathrm{C}$ for 24 hours (Figure 1). Single isolated colonies were inoculated onto Cetrimide agar, MacConkey agar, and Eosin Methylene Blue agar $\left(37^{\circ} \mathrm{C}\right.$ for $\left.24 \mathrm{~h}\right)$. Colonies that were blue greenish on Cetrimide agar were identified as $P$. aeruginosa. The colonies with large, pink, gummy, mucoid on MacConkey agar and sheened with green metallic color on Eosin Methylene Blue agar were identified as $K$. pneumoniae and E. coli, respectively. Moreover, on MacConkey plates, E. coli showed flat, dry, pink colonies with a surrounding darker pink area of precipitated bile salts. The colonies were subcultured to obtain pure cultures.

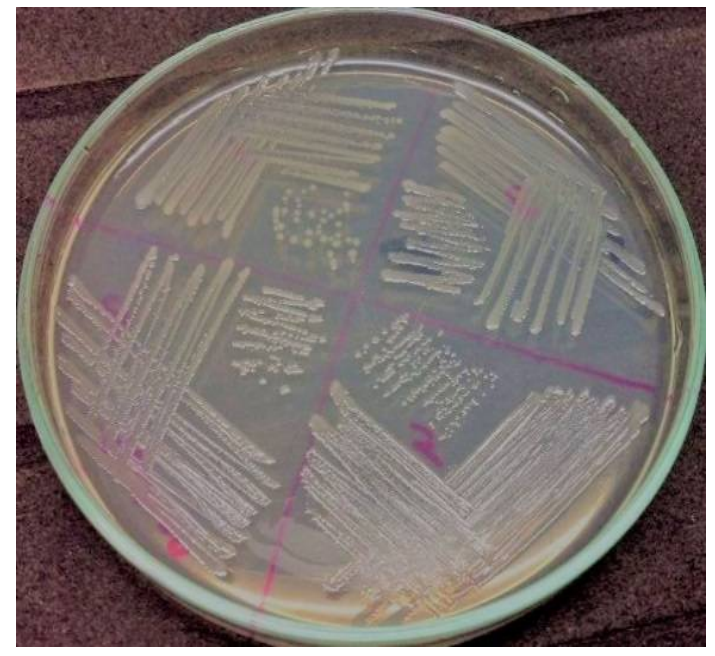

Figure 1. Colonies on nutrient agar plate.

Gram staining. The standard procedures were followed to perform Gram staining for all forty isolates. A thin smear was prepared and the slide was air dried. Heat fixation was done by passing the slide over a flame. The smear was flooded with crystal violet and was allowed to stand for 45 seconds. The crystal violet was washed away with a gentle stream of water followed by adding Grams iodine (mordant). The smear was decolorized by adding $95 \%$ alcohol to the slide and was washed away after 10 seconds. Finally, the slide was flooded with the counterstain 
safranin and allowed to stand for 1 minute. The slide was washed, dried and was ready to be visualized under bright field microscope.

Biochemical tests. The isolates were further distinguished by using traditional biochemical tests; Kligler's iron agar test, Motility-indole-ornithine test, citrate utilization test, Methyl Red-Voges Proskauer test, indole test, and catalase test.

Catalase test. A bacterial colony was transferred onto a sterile glass slide using a sterile wooden stick followed by adding a drop of $3 \%$ hydrogen peroxide on the colony. Formation of air bubble indicated catalase positive and no bubble production indicated catalase negative. Staphylococcus aureus-ATCC 33592 and Enterococcus faecalis-ATCC 29212 were used as a positive and negative control, respectively.

Kligler's iron agar (KIA) test. All bacterial isolates were tested for KIA test to study the mode of dextrose utilization in oxidative/fermentative test. Bacterial isolate was stabbed into the butt and streaked onto the slant of KIA medium and incubated for 24 hours at $37^{\circ} \mathrm{C}$. The ability of bacteria to ferment glucose and/or lactose, and production of hydrogen sulfide $\left(\mathrm{H}_{2} \mathrm{~S}\right)$ or other gases were the attributes for the test. On KIA, a yellow slant indicated that the organism fermented lactose and yellow butt indicated glucose utilization. Black precipitation in the butt indicated $\mathrm{H}_{2} \mathrm{~S}$ production. Production of gases other than $\mathrm{H}_{2} \mathrm{~S}$ was indicated either by cracks or bubbles in the media or by the media being pushed away from the bottom of the tube. S. aureus ATCC 25923 was used as positive control.

Motility-indole-ornithine (MIO) test. A positive motility test was indicated by a diffuse cloud of growth away from the line of inoculation. A positive test for indole was denoted when a pink to the red color band was formed at the top of the medium after addition of Kovacs reagent and yellow color denoted a negative indole test. A positive test for ornithine was denoted by a dark, turbid purple color in the medium. A yellow color throughout the medium denoted a negative ornithine result.
Citrate test. Simmons citrate agar test was performed to determine the ability of organisms to utilize citrate as a carbon source. The organism was citrate positive if medium turned to blue color and if the media retained its green color, the bacteria was detected as citrate negative. S. aureus ATCC 25923 was used as a positive control and E. coli ATCC 25922 was used as a negative control.

Methyl Red (MR) test. The test was performed by inoculating a colony of the test organism in $5 \mathrm{ml}$ of methyl red medium. After 24 hours incubation at $37^{\circ} \mathrm{C}$, a drop of methyl red solution was added. The tubes were then shaken and examined. A distinct bright red color indicated MR positive test and yellow color indicated MR negative test.

Voges Proskauer (VP) test. For VP test, after inoculation and incubation of the culture tube for 24 hours at $37^{\circ} \mathrm{C}, 0.6 \mathrm{ml}$ of $5 \%$ alpha-naphthol was added, followed by addition of $0.2 \mathrm{ml}$ of $40 \% \mathrm{KOH}$. After gentle shaking, the tubes were kept undisturbed for 10-15 minutes. Red color indicated VP positive and presence of no color indicated VP negative.

Indole test. The isolates were inoculated into 3 $\mathrm{ml}$ of the peptone water. The vials were incubated for 24 hours at $37^{\circ} \mathrm{C}$. Three to four drops of Kovac's reagent was added and the color was noted. Formation of rose-purple color indicated a positive reaction.

Antimicrobial sensitivity testing. The KirbyBauer disk diffusion method was used to determine antibiotic sensitivity profile of 40 isolates using 20 antibiotics which are commonly prescribed against RTIs in Bangladesh. The antibiotics were chosen on the basis of their importance in treating infections. For sensitivity testing, the isolates were cultured onto Mueller-Hinton agar (Merck, Germany) plates. The standard antibiotic discs were placed on the surface of the inoculated agar plates followed by incubation at $37^{\circ} \mathrm{C}$ for 24 hours in an aerobic condition. The antibiotic disks used in the study included amoxicillin (25 $\mu \mathrm{g})$, amikacin $(30 \mu \mathrm{g})$, amoxiclav $(30 \mu \mathrm{g})$, cefixime $(5 \mu \mathrm{g})$, cefotaxime $(30 \mu \mathrm{g})$, ceftazidime (30 $\mu \mathrm{g})$, ceftriaxone $(30 \mu \mathrm{g})$, cefuroxime $(30 \mu \mathrm{g})$, cephalexin $(30 \mu \mathrm{g})$, cephradine $(15 \mu \mathrm{g})$, ciprofloxacin 
(5 $\mu \mathrm{g})$, colistin $(15 \mu \mathrm{g})$, gentamycin $(30 \mu \mathrm{g})$, imipenem $(10 \mu \mathrm{g})$, meropenem $(10 \mu \mathrm{g})$, cotrimoxazole $(30 \mu \mathrm{g})$, piperacillin-tazobactam (30 $10 \mu \mathrm{g})$, nitrofurantoin $(300 \mu \mathrm{g})$, nalidixic acid (30 $\mu \mathrm{g})$, levofloxacin $(5 \mu \mathrm{g})$ (Merck KGaA, Darmstadt, Germany) per disc. The plates were observed for the zone of inhibition after 24 hours (Figure 3). Antibiotic sensitivity of the isolates was determined by measuring the diameter of each zone of inhibition around each disc compared with a standard chart. ${ }^{12}$ The zone diameter for an individual antibiotic was translated into susceptibility, intermediate or resistant categories according to a guideline of Fluka-SigmaAldrich, 2015. ${ }^{12,13}$

\section{RESULTS AND DISCUSSION}

Bacterial isolates. A total of forty bacterial isolates from RTIs were examined by cultural and biochemical testing. The findings revealed that 17 were $K$. penumoniae, 12 were $E$. coli, and 11 were $P$. aeruginosa. The $P$. aeruginosa produced bluish green colonies on cetrimide agar plates and $K$. pneumoniae produced pink mucoid gummy colonies on MacConkey plates (Figure 2A). E.coli was identified by the green metallic sheen on Eosin Methylene Blue media and on MacConkey plate they showed flat, dry, pink colonies with a surrounding darker pink area of precipitated bile salts (Figure 2B).

For the conformational identity of the clinical isolates, a number of biochemical tests were performed. For KIA, E07, and E11 which showed an alkaline reaction in the butt. However, all the other $E$. coli showed an acidic reaction in both slant and butt of KIA medium, a reaction indicative of both lactose and glucose fermentation (Table1). All of the $P$. aeruginosa isolates showed red slant and butt that indicated neither glucose nor lactose was fermented (Table 3). For K. pneumoniae, both the butt and the slant were yellow meant that both glucose and lactose was fermented (Table 2). For all of the isolates in any case no $\mathrm{H}_{2} \mathrm{~S}$ and other gas was produced.

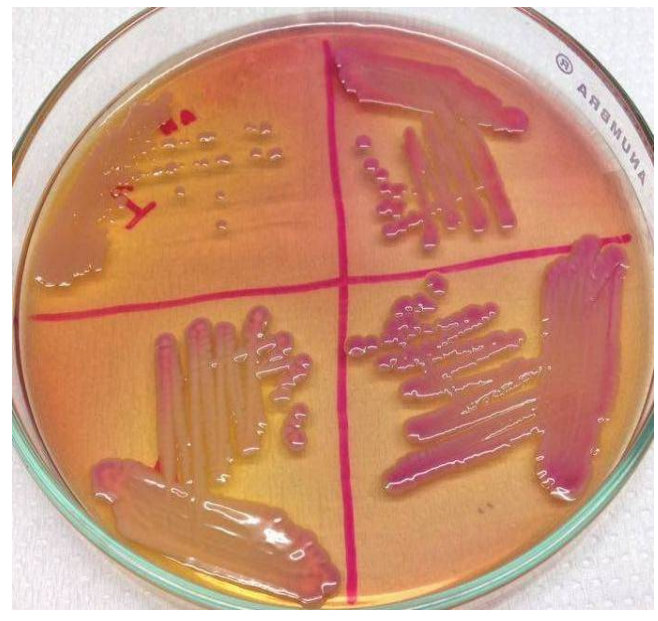

A

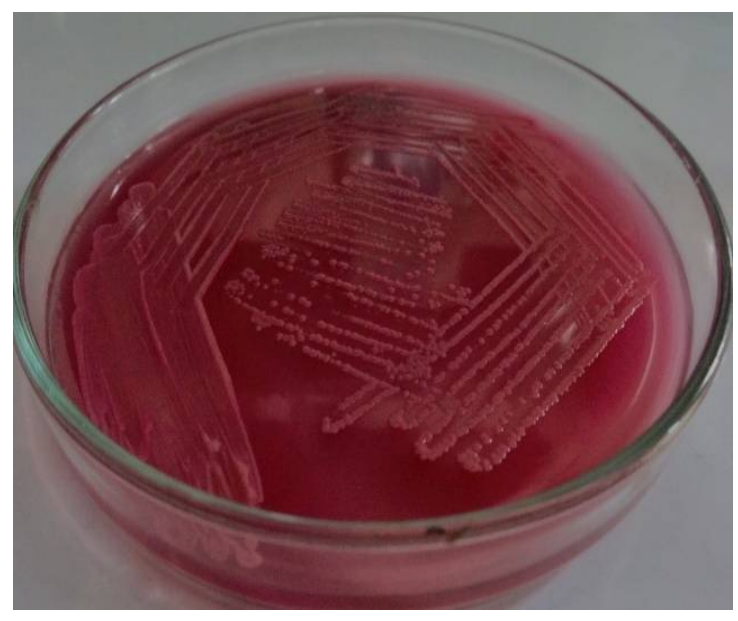

B

Figure 2. K. pneumoniae (A) and E.coli (B) on MacConkey agar plate.

In MIO media, all the E.coli isolates showed motility in the form of cloudy growth and the ornithine decarboxylation test was positive by the purple coloration of the media except for E03, E04, E07 and E11. A positive test for indole was observed, after addition of Kovac's Reagent and a red color band was formed on top of the medium (Table 1). Isolates of $K$. pneumoniae showed no motility in
MIO medium, and showed negative indole and ornithine decarboxylation reaction (Table 2). All the isolates of $P$. aeruginosa, showed positive motility but a negative indole and ornithine test (Table3).On citrate media, all the E.coli isolates were unable to utilize citrate and the color of the media remained green (Table 1). K. pneumoniae and $P$. aeruginosa 
gave a positive result and the media turned into deep prussian blue (Table 2, Table 3).

In MR-VP reaction, interestingly, all the $E$. coli isolates gave MR positive and VP negative result and the $K$. pneumoniae gave the MR negative and VP positive result. And for $P$. aeruginosa both MR-VP were negative. All of the E. coli, K. pneumoniae, $P$. aeruginosa species were catalase positive.

Table 1. Biochemical characteristics of Escherichia coli clinical isolates.

\begin{tabular}{|c|c|c|c|c|c|c|}
\hline SI & $\begin{array}{l}\text { KIA } \\
\text { (butt/slant/gas } \\
\text { production/H2S) }\end{array}$ & $\begin{array}{c}\text { MIO } \\
\text { (motility/indole/ } \\
\text { ornithine) }\end{array}$ & Citrate test & $\begin{array}{l}\text { MR- } \\
\text { VP }\end{array}$ & Indole test & Catalase test \\
\hline E01 & acid / acid / + / - & $+1+1+$ & - & $+1-$ & + & + \\
\hline E02 & acid / acid / + / - & $+1+1+$ & - & $+1-$ & + & + \\
\hline E03 & acid / acid / + / - & $+1+1-$ & - & $+1-$ & + & + \\
\hline E04 & acid / acid / + / - & $+1+1-$ & - & $+1-$ & + & + \\
\hline E05 & acid / acid / + / - & $+1+1+$ & - & $+1-$ & + & + \\
\hline E06 & acid / acid / + / - & $+1+1+$ & - & $+1-$ & + & + \\
\hline E07 & alkaline/acid/+/- & $+1+1-$ & - & $+1-$ & + & + \\
\hline E08 & $\operatorname{acid} /$ acid / + / - & $+1+1+$ & - & $+1-$ & + & + \\
\hline E09 & acid / acid / + / - & $+1+1+$ & - & $+1-$ & + & + \\
\hline E10 & acid / acid / + / - & $+/+1+$ & - & $+1-$ & + & + \\
\hline E11 & alkaline/acid/+/- & $+1+1-$ & - & $+1-$ & + & + \\
\hline E12 & acid / acid / + / - & $+/+1+$ & - & $+1-$ & + & + \\
\hline
\end{tabular}

Table 2. Biochemical characteristics of Klebsiella pneumoniae clinical isolates.

\begin{tabular}{|c|c|c|c|c|c|c|}
\hline SI & $\begin{array}{l}\text { KIA } \\
\text { (butt/slant/gas } \\
\text { production/ } / \mathrm{H}_{2} \mathrm{~S} \text { ) }\end{array}$ & $\begin{array}{c}\text { MIO } \\
\text { (motility/indole/ } \\
\text { ornithine) }\end{array}$ & Citrate test & MR-VP & $\begin{array}{c}\text { Indole } \\
\text { test }\end{array}$ & $\begin{array}{c}\text { Catalase } \\
\text { test }\end{array}$ \\
\hline K01 & alkaline/alkaline/+/- & $-1-1-$ & + & $-1+$ & - & + \\
\hline K02 & alkaline/alkaline/+/- & $-1-1-$ & + & $-/+$ & - & + \\
\hline K03 & alkaline/alkaline/+/- & $-1-1-$ & + & $-/+$ & - & + \\
\hline K04 & alkaline/alkaline/+/- & $-1-1-$ & + & $-/+$ & - & + \\
\hline K05 & alkaline/alkaline/+/- & $-1-1-$ & + & $-/+$ & - & + \\
\hline K06 & alkaline/alkaline/-/- & $-1-1-$ & + & $-1+$ & - & + \\
\hline K07 & alkaline/alkaline/+/- & $-1-1-$ & + & $-1+$ & - & + \\
\hline K08 & alkaline/alkaline/+/- & $-1-1-$ & + & $-/+$ & - & + \\
\hline K09 & alkaline/alkaline/+/- & $-1-1-$ & + & $-1+$ & - & + \\
\hline K10 & alkaline/alkaline/+/- & $-1-1-$ & + & $-/+$ & - & + \\
\hline K11 & alkaline/alkaline/+/- & $-1-1-$ & + & $-/+$ & - & + \\
\hline K12 & alkaline/alkaline/+/- & $-1-1-$ & + & $-/+$ & - & + \\
\hline K13 & alkaline/alkaline/+/- & $-1-1-$ & + & $-/+$ & - & + \\
\hline K14 & alkaline/alkaline/+/- & $-1-1-$ & + & $-/+$ & - & + \\
\hline K15 & alkaline/alkaline/-/- & $-1-1-$ & + & $-/+$ & - & + \\
\hline K16 & alkaline/alkaline/+/- & $-1-1-$ & + & $-/+$ & - & + \\
\hline K17 & alkaline/alkaline/+/- & $-1-1-$ & + & $-/+$ & - & + \\
\hline
\end{tabular}


Table 3. Biochemical characteristics of Pseudomonas aeruginosa clinical isolates.

\begin{tabular}{|c|c|c|c|c|c|c|}
\hline SI & $\begin{array}{l}\text { KIA } \\
\text { (butt/slant/gas production/ } \mathrm{H}_{2} \mathrm{~S} \text { ) }\end{array}$ & $\begin{array}{c}\text { MIO } \\
\text { (motility/indole/ } \\
\text { ornithine) }\end{array}$ & Citrate test & $\begin{array}{l}\text { MR- } \\
\text { VP }\end{array}$ & $\begin{array}{c}\text { Indole } \\
\text { test }\end{array}$ & $\begin{array}{c}\text { Catalase } \\
\text { test }\end{array}$ \\
\hline P01 & alkaline/alkaline/-/- & $+1-1-$ & + & $-1-$ & - & + \\
\hline $\mathrm{P} 02$ & alkaline/alkaline/-/- & $+1-1-$ & + & $-1-$ & - & + \\
\hline $\mathrm{P} 03$ & alkaline/alkaline/-/- & $+1-1-$ & + & $-1-$ & - & + \\
\hline P04 & alkaline/alkaline/-/- & $+1-1-$ & + & $-1-$ & - & + \\
\hline P05 & alkaline/alkaline/-/- & $+1-1-$ & + & $-1-$ & - & + \\
\hline P06 & alkaline/alkaline/-/- & $+1-1-$ & + & $-1-$ & - & + \\
\hline P07 & alkaline/alkaline/-/- & $+1-1-$ & + & $-1-$ & - & + \\
\hline P08 & alkaline/alkaline/-/- & $+1-1-$ & + & $-/-$ & - & + \\
\hline P09 & alkaline/alkaline/-/- & $+1-1-$ & + & $-1-$ & - & + \\
\hline P10 & alkaline/alkaline/-/- & $+1-1-$ & + & $-/-$ & - & + \\
\hline P11 & alkaline/alkaline/-/- & $+1-1-$ & + & $-1-$ & - & + \\
\hline
\end{tabular}

Demographic features of RTI patients. During the 3 months period, 40 respiratory samples were processed for culture and sensitivity testing. Sputum samples from patients of all age groups (from 6 months to $>70$ years) and both sexes were processed. The results are presented in Table 3. The results showed that RTIs was $15 \%$ more among male than female patients with no significant gender variation (p-value, <0.578) (Table 4). Among female patients, the highest occurrence of RTI was in the age group 46-70 years, which was 59\% of the total female patients. It was similar for men with $43 \%$. The age group of 20-45 years female also had a high ratio of $35 \%$. On the other hand, while $>70$ age group RTI patients were only $5 \%$ in the females, it was significantly higher among males which were $30 \%$. Younger patients (0-19 years) were higher in males $(17 \%)$. In contrary, there was no female patient in this age group among the 40 patients infected with bacteria.

Antibiotic sensitivity pattern of bacterial isolates. The study analyzed individual antibiotic sensitivity and overall sensitivity among different classes of antibiotics. It was shown that all three species of E. coli, $K$. pneumoniae and $P$. aeruginosa were highly sensitive $(80-100 \%)$ to carbapenem antibiotics; meropenem, imipenem, amikacin and colistin. Next sensitive antibiotic class was aminoglycosides with average $60 \%$ ranging from 55$69 \%$ followed by sulfadrug $(42.5 \%)$ and fluoroquinolone (average; $40.83 \%$, range; $33-53 \%$ ). The surprising sensitivity resulted for cephalosporin antibiotics (average; 31\%, range; 24-40\%) and for penicillin antibiotics (average; 22\%, range; 0-30\%. (Table 5, Figure 4).

Table 4. Age and sex wise distribution of RTI patients.

\begin{tabular}{lllll}
\hline $\begin{array}{l}\text { Age } \\
(\text { Years })\end{array}$ & $\begin{array}{l}\text { Male } \\
(\mathrm{N}=23)\end{array}$ & $\begin{array}{l}\text { Female } \\
(\mathrm{N}=17)\end{array}$ & $\begin{array}{l}\text { Culture }(+) \\
\text { Males }(\%)\end{array}$ & $\begin{array}{l}\text { Culture }(+) \\
\text { Females }(\%)\end{array}$ \\
\hline $0-19$ & 4 & 0 & 10 & 0 \\
$20-45$ & 2 & 6 & 5 & 15 \\
$46-70$ & 10 & 10 & 25 & 25 \\
$>70$ & 7 & 1 & 17.5 & 2.5 \\
\hline Total & $\mathrm{N}=40$ & & $57.5 \%$ & $42.5 \%$ \\
\hline p-value & & \multicolumn{5}{c}{0.578} \\
\hline
\end{tabular}


Table 5. Antibiotic sensitivity and resistance pattern of RTI patient to different antibiotic classes.

\begin{tabular}{lllllllll}
\hline Antibiotic class & \multicolumn{4}{c}{ Sensitivity } & \multicolumn{5}{c}{ Resistance } \\
\cline { 2 - 7 } & Overall & $\% E$ & $\% \mathrm{P}$ & $\% \mathrm{~K}$ & $\% \mathrm{E}$ & $\% \mathrm{P}$ & $\% \mathrm{~K}$ & Overall \\
\hline Penicillin & 21.67 & 30 & 0 & 29 & 64 & 67 & 59 & 62.5 \\
Cephalosporin & 30.71 & 24 & 25 & 40 & 76 & 73 & 58 & 67.5 \\
Fluoroquinolone & 40.83 & 53 & 39 & 33 & 47 & 55 & 59 & 54.17 \\
Carbapenem & 86.67 & 100 & 82 & 80 & 0 & 18 & 20 & 13.33 \\
Aminoglycosides & 60 & 67 & 55 & 59 & 33 & 46 & 41 & 40.0 \\
Sulfa drug & 42.5 & 17 & 0 & 88 & 58 & 100 & 12 & 51.63 \\
Average & 47.06 & 46 & 35 & 49 & 51 & 58 & 48 & 48.18 \\
p-value based on & 0.00 & 0.00 & 0.00 & 0.00 & 0.00 & 0.00 & 0.00 & 0.00 \\
Pearson Chi ${ }^{2}$ test & & & & & & & & \\
\hline
\end{tabular}

E.coli $(\mathrm{E})$, Pseudomonas aeruginosa $(\mathrm{P})$, Klebsiella pneumoniae $(\mathrm{K})$

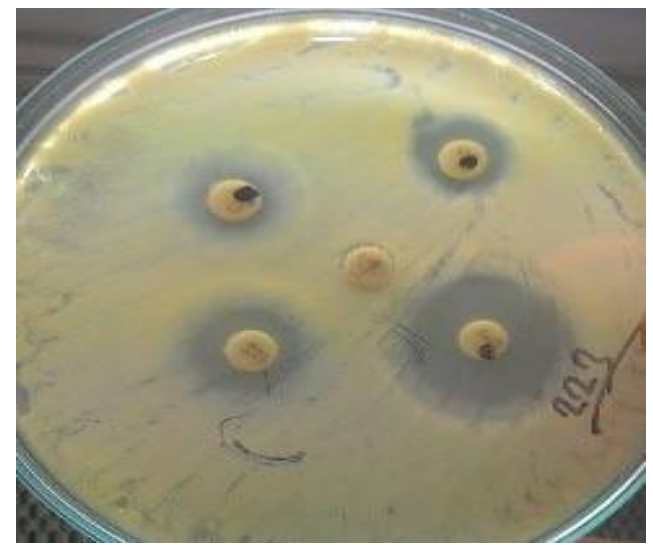

Figure 3. Antibiotic Sensitivity Test.

$100 \%$ E. coli were highly sensitive against meropenem, imipenem, amikacin, and colistin. E. coli also showed very good sensitivity against nitrofurantoin (78\%) and levofloxacin (89\%). However, only $24 \%$ E. coli showed sensitivity to cephalosporin as an average (Figure 4). In case of $K$. pneumoniae, nearly $80 \%$ were sensitive to meropenem, imipenem, amikacin, colistin, cotrimoxazole, and gentamycin. However, the lower sensitivity observed for cephalosporin antibiotics 20$40 \%$ (Table 5).

No $P$. aeruginosa were $100 \%$ sensitive to any antibiotics. The highest sensitivity (about 7585\%)was shown bymeropenem, imipenem, colistin and amikacin and nearly $60 \%$ sensitivity observed for gentamycin, nalidixic acid, ciprofloxacin and ceftazidime (Figure 4).
Antibiotic resistance pattern of RTI patients. On average $48 \%$ of tested antibiotics became resistant the pathogens irrespective of their types, which surpassed sensitive antibiotics (47\%) against RTIs. There were significant differences ( $\mathrm{p}$-value $<0.000)$ in the resistance pattern $(13-67 \%)$ among antibiotic classes for each type of pathogen (Table 5). $P$. aeruginosa was of highest ranked to show resistance against varieties of selected antibiotics. This species showed $100 \%$ resistance against amoxicillin and showed resistance (40-55\%) against amoxiclav, cotrimoxazole, ciprofloxacin, piperacillin/ tazobactam, and cefotaxime. The antibiotics against which $>70 \%$ P. aeruginosa proved resistant were ceftriaxone, cefotaxime, cefuroxime, cephalexin, cefixime, cephradine, levofloxacin andnitrofurantoin (Figure 4).

E. coli showed $100 \%$ resistance only against amoxicillin, however, 60-85 \% E. coli showed resistance against many of cephalosporin antibiotics such as cefuroxime, ceftriaxone, cephradine, cefixime, cephalexin, ceftazidime, and aminoglycoside antibiotics, gentamycin and sulfa drug cotrimoxazole (Figure 4). These results are threatening for the treatment of $E$. coli mediated RTI. On the other hand, resistance pattern of $K$. pneumoniae was similar with E.coli in case of amoxicillin (100\%) and cephalosporins (60-80\%). However, the exception was for nitrofurantoin and 
levofloxacin with $77 \%$ and $82 \%$ resistance, respectively (Figure 4).

Relationship between patients' age and antibiotic sensitivity. The Kruskal-Wallis equalityof-populations rank test was applied to determine whether antibiotic sensitivity response varied with different age group patients. The analysis revealed that there was no statistically significant variation in the sensitivity response (p-value $<0.520)$ and resistance ( $\mathrm{p}$-value <0.351) pattern of antibiotics among different-aged RTI patients (Table 6). Overall 9 out of 20 antibiotics $(45 \%)$ were effective and 10 out of 20 antibiotics were ineffective for all aged RTIs patients.

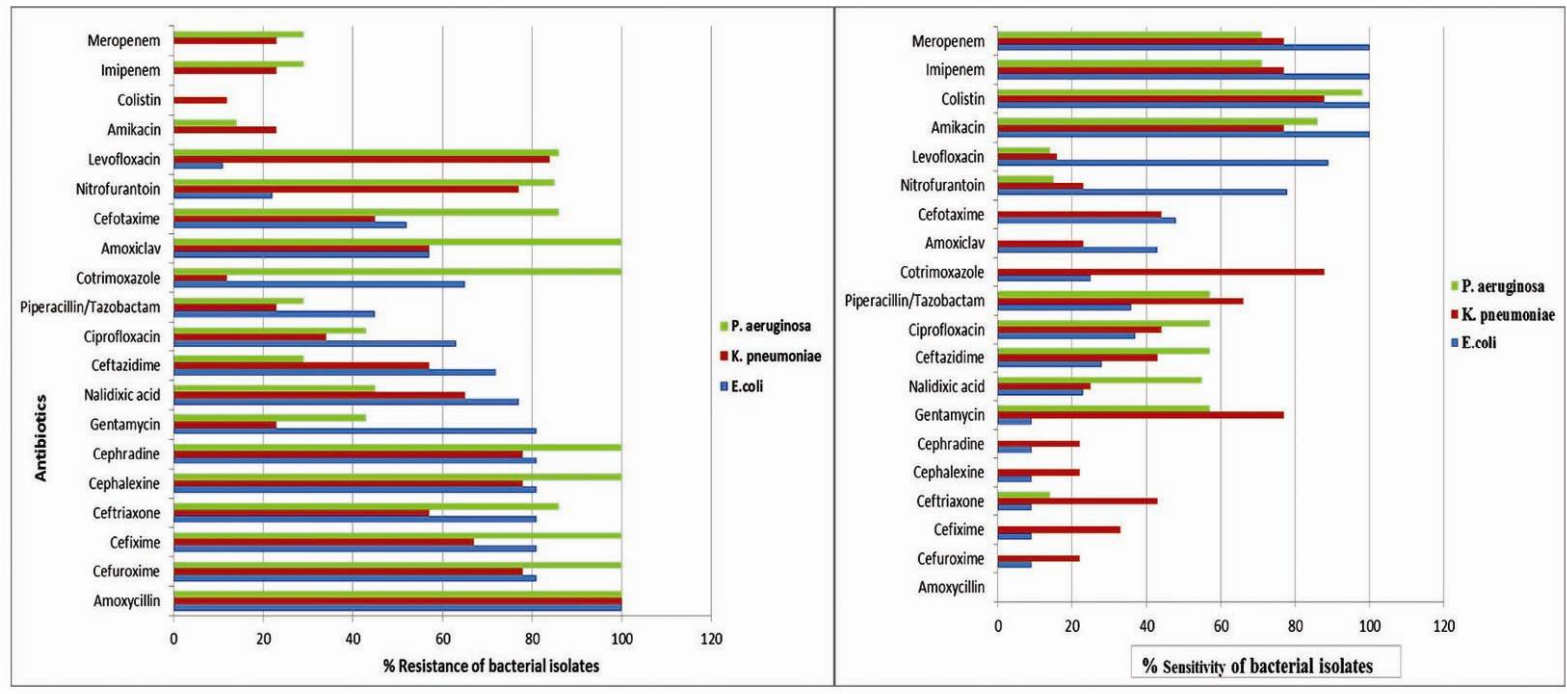

Figure 4. Antibiotic sensitivity pattern of bacteria isolated from RTI patients.

Table 6. Relationship among RTI patient age with sensitivity and resistance pattern of antibiotics using Kruskal-Wallis equality-ofpopulations rank test.

\begin{tabular}{|c|c|c|c|c|c|c|c|}
\hline Age (year) & $\begin{array}{c}\text { No. of } \\
\text { pathogen }\end{array}$ & $\begin{array}{c}\text { Sensitive } \\
\text { antibiotics } \\
\text { (mean) }\end{array}$ & SD & p-value & $\begin{array}{c}\text { Resistant } \\
\text { antibiotics } \\
\text { (mean) }\end{array}$ & $\mathrm{SD}$ & p-value \\
\hline$<=19$ & 5 & 9.2 & 1.9 & \multirow{5}{*}{0.520} & 10.2 & 2.1 & \multirow{5}{*}{0.351} \\
\hline $20-45$ & 8 & 9.1 & 1.95 & & 10 & 2.82 & \\
\hline $46-70$ & 18 & 9.1 & 2.1 & & 9.9 & 2.3 & \\
\hline$>70$ & 9 & 7.8 & 1.9 & & 11.4 & 2.12 & \\
\hline Total & $\mathrm{N}=40$ & 8.8 & & & 10.37 & & \\
\hline
\end{tabular}

Implication of findings. AR of pathogenic bacteria has become a crisis serious, and antibiotics misuse has been identified as a key driver of this crisis. $^{2} \quad$ RTIs are considered as the frequently occurred human infections and antibiotic misuse against RTIs is more common in Bangladesh as well as in the world. The infections including RTIs caused by the resistant strains are more likely to enhance hospitalizations, failure of treatment and mortality. ${ }^{5}$
Not only having selective pressure but also bacteria themselves have the ability to change their gene cassette and increase immunity against antibiotics. These two phenomena may explain the rapid change in the modality of antibiotic resistance among pathogenic bacteria. ${ }^{6}$

In the present study, three types of bacterial isolates were obtained and characterized among RTI infected patients. They were E. coli, K. pneumonia 
and $P$. aeruginosa. Among study samples, RTIs distribution were $15 \%$ more among male than female patients with no significant gender variation. This finding was congruent with a study conducted in Spain found that male were $24 \%$ (62\% male vs. $38 \%$ female) more likely to have RTIs caused by resistant bacteria. ${ }^{14}$ The age distribution revealed the highest occurrence (59\%) of RTIs within female patients under age group 46-70 years compared to male (43\%). On the other hand, above 70 years, only 5\% RTI patients were females than $30 \%$ in males. $17 \%$ males and no female patients within aged 0-19 years found in the study samples. In contrast, among under-five age group children and over 65 age group people, acute RTIs are the most common reasons of illness and death. ${ }^{15}$

Antibiogram reports showed that overall $47 \%$ (out of 20) antibiotics were sensitive, whereas $48 \%$ were resistant to pathogens. However, there were significant sensitivity (22-87\%) differences among antibiotic classes ( $p$-value $<0.000$ ) for each type of pathogen. It was alarming that $18 \% P$. aeruginosa and $20 \% \mathrm{~K}$. pneumoniae showed carbapenem resistance and $67 \%$ cephalosporin antibiotics were resistant. However, E. coli showed very good sensitivity to nitrofurantoin (78\%) and levofloxacin (89\%) and K. pneumoniae to cotrimoxazole (88\%), gentamycin (77\%), and piperacillin-tazobactam (66\%). This trend might be due to excessive use of cephalosporin antibiotics against RTIs. This is supported by the fact that prescribers choose cephalosporin antibiotics to avoid patients' risk of failure to low spectrum antibiotic prescription in RTIs in Bangladesh. ${ }^{10}$ The higher cephalosporin resistance $(67 \%)$ in the present study were similar with the data of cephalosporin resistance among $E$. coli isolates in Pakistan (90\%), India (83\%) and Russia (77\%). ${ }^{16} 100 \%$ sensitivity of $E$. coli isolates to carbapenem antibiotics observed in this study however, in India, 11\% of 408 isolates were resistant against them. ${ }^{17}$ The fluoroquinolones resistance were comparatively lower in Bangladesh for E.coli isolates (47\%) compared to India (84\%) and for $P$. aeruginosa (54\%) compared to Romania (66\%), India (55\%), Belarus (86\%) and South Africa
$(35 \%) .{ }^{17}$ However, E. coli resistance is significantly lower among Australia, United Kingdom, South Africa and United States. ${ }^{16,17}$

The results of the study have significance at the point of view RTI patients still may have a better response to some fluoroquinolone, aminoglycoside and cotrimoxazole antibiotics, if patients are infected with E. coli and or K. pneumoniae. This information is valuable for RTIs management especially to reduce blind prescription of 3rd to 4th generation cephalosporin antibiotics in Bangladesh. Prescribers should change their attitudes of malpractice of antibiotics in RTIs with an urgent priority. ${ }^{18}$ Moreover, it needs further investigations on large scale to validate and upgrade results of antibiotic sensitivity in RTIs. The strength of the study is exploring the current antibiotic sensitivity pattern to RTI infection which would be very informative to the Bangladeshi prescribers. The study recommends them to prescribe nitrofurantoin, gentamycin, cotrimoxazole, levofloxacin, piperacillin-tazobactam in case of bacterial RTIs rather than broad-spectrum antibiotics like cephalosporin. Prescribers should change their behavior of unnecessary and over prescribing attitude of broad-spectrum cephalosporin antibiotics in RTIs to slow down the pace of over rising AR in Bangladesh. ${ }^{18}$

\section{CONCLUSIONS}

K. pneumoniae, E. coli, and P. aeruginosaare were found common bacterial pathogens in RTIs. The meropenem, imipenem, amikacin, colistin, and piperacillin-tazobactam are high sensitive antibiotics to bacterial RTIs. Nitrofurantoin, levofloxacin, cotrimoxazole, gentamycin and piperacillintazobactam antibiotics are better prescribing options to treat RTIs as recommended to prescribers in Bangladesh.

\section{REFERENCES}

1. World Health Organization (WHO). 2014. Antimicrobial resistance: global report on surveillance: World Health Organization. http://apps.who.int/ 
2. El-Mahmood, A., Isa, H., Mohammed, A. and Tirmidhi, A. 2010. Antimicrobial susceptibility of some respiratory tract pathogens to commonly used antibiotics at the Specialist Hospital, Yola, Adamawa State, Nigeria. J. Clin. Med. Res. 2, 135-142.

3. O'Neill, J. 2015. Tackling a crisis for the health and wealth of nations. The Review on Antimicrobial Resistance. https://amr-review.org

4. Jones, R. N. and Pfaller, M. A. 1998. Bacterial resistance: a worldwide problem. Diagn. Microbiol. Infect. Dis. 31, 379388.

5. Ball, P., Baquero, F., Cars, O., File, T., Garau, J. and Klugman, K. T. 2002. Antibiotic therapy of community respiratory tract infections: strategies for optimal outcomes and minimized resistance emergence. J. Antimicrob Chemoth. 49, 31-40.

6. Cantas, L., Shah, S. Q., Cavaco, L. M., Manaia, C., Walsh, F., Popowska, M. and Sørum, H. 2013. A brief multidisciplinary review on antimicrobial resistance in medicine and its linkage to the global environmental microbiota. Front Microbiol. 4.

7. Martínez-González, N. A., Coenen, S., Plate, A., Colliers, A., Rosemann, T.,Senn, O. and Neuner-Jehle, S. 2017. The impact of interventions to improve the quality of prescribing and use of antibiotics in primary care patients with respiratory tract infections: a systematic review protocol. BMJ Open. 7, e016253.

8. Baqui, A. H., Rahman, M., Zaman, K., El Arifeen, S., Chowdhury, H. R., Begum, N. and Santosham, M. 2007. A population-based study of hospital admission incidence rate and bacterial aetiology of acute lower respiratory infections in children aged less than five years in Bangladesh. J. Health Popul. Nutr. 25, 179.

9. Alter, S. J., Vidwan, N. K., Sobande, P. O., Omoloja, A. and Bennett, J. S. 2011. Common childhood bacterial infections. Curr. Probl. Pediatr. Adolesc. Health Care. 41, 256-283.
10. Rahman, M. S. and Huda, S. 2014. Antimicrobial resistance and related issues: An overview of Bangladesh situation. Bangladesh J. Pharmacol. 9, 218-224.

11. Gradwohl, R.B.H., Sonnenwirth, A.C. and Jarett, L. 1980. Gradwohl's clinical laboratory methods and diagnosis: Mosby Available from http://agris.fao.org/agris-search/search

12. Fluka-Sigma-Aldrich. 2015. Antimicrobial susceptibility test discs. Available from:https://www.sigmaaldrich.com/content/ dam/sigma- ldrich/docs/Sigma/General_Information /antimicrobial suscept_discs_leaflet.pdf accessed 25 Aug 2016.

13. Poddar, S.K., Saqueeb, N. and Rahman, S.M.A. 2016. Synthesis and biological evaluation of 2-methyl1Hbenzimidazole and 1H-benzimidazol-2-yl-methanol. Dhaka Univ. J. Pharm. Sci. 15, 83-87.

14. Hurtado, S.E.J., Fernandez, G.M.J., Rodriguez, C.J., Aranguena, G.L. and Angulo, G.G.E. 2017. Lower respiratory tract infections and espectrum beta lactamase resistant microorganisms. Bacterial respiratory infections. Ann. Am. Thorac. Soc. A3940-A3940.

15. Ramani, V.K., Pattankar. J. and Puttahonnappa, S.K. 2016. Acute respiratory infections among under-five age group children at urban slums of Gulbarga city: A longitudinal study. J. Clin. Diagn. Res.10, LC08.

16. The Center for Disease Dynamics, Economics (CDDEP). 2017. Resistance map: Antibiotic Resistance. https://resistancemap.cddep.org/

17. Promite, S., Saha, S.K. and Roy, P.K. 2017. Emerging Antibiotic Resistance and Plasmid DNA in Escherichia coli Isolated from Respiratory Tract Infections in Bangladesh. Open Access J. Trans. Med. Res. 1, 00011.

18. Saha, S.K. and Promite, S. 2017. Factors influencing clinician's antibiotic prescribing behaviors (APB) in Bangladesh: An in-depth review using COMB Model. Open Access J. Trans. Med. Res.1, 00019. 